\title{
Use of an Expedited Review Tool to Screen for Prior Diagnostic Error in Emergency Department Patients
}

J. Hudspeth'; R. El-Kareh²; G. Schiff ${ }^{3}$

${ }^{1}$ Department of Medicine, Boston University, Boston, MA, United States; ${ }^{2}$ Department of Medicine, University of California, San Diego, CA, United States; ${ }^{3}$ Department of Medicine, Brigham and Women's Hospital, Boston, MA, United States

\section{Keywords}

Monitoring and surveillance, error management and prevention, emergency and disaster care, data processing, human-computer interaction, diagnostic error

\section{Summary}

Objective: Missed diagnoses are an important area of care quality resulting in significant morbidity and mortality. Determination of rates and causes has been limited by difficulties in screening, including the effort of manual chart review. We developed and tested a semi-automated review tool to expedite screening for diagnostic errors in an electronic health record (EHR).

Methods: We retrospectively reviewed patients seen in the emergency department (ED) of a teaching hospital over 31 days, using an automated screen to identify those with a prior in-system visit during the 14 days preceding their ED visit. We collected prior and subsequent notes from the institution's EHR for these cases, then populated a specially designed relational database enabling rapid comparison of prior visit records to the sentinel ED visit. Each case was assessed for potential missed or delayed diagnosis, and rated by likelihood as "definite, probable, possible, unlikely or none."

Results: A total of 5066 patient encounters were screened by a clinician using the tool, of which $1498(30 \%)$ had a clinical encounter within the preceding 14 days. Of these, 37 encounters $(2.6 \%$ of those reviewed) were "definite" or "probable" missed diagnoses. The rapid review tool took a mean of 1.9 minutes per case for primary review, compared with 11.2 minutes per case for reviews without the automated tool.

Conclusions: Diagnostic errors were present in a significant number of cases presenting to the ED after recent healthcare visits. An innovative review tool enabled a substantially increased efficiency in screening for diagnostic errors.

\section{Correspondence to:}

James Hudspeth, MD

Section of General Internal Medicine

801 Massachusetts Ave, Crosstown Center 2nd floor

Boston, MA 02118

Tel.: 617-984-9212

Fax: 888-958-5409

Email: jahudspe@bu.edu
Appl Clin Inform 2015; 6: 619-628

http://dx.doi.org/10.4338/ACl-2015-04-RA-0042

received: April 22, 2015

accepted in revised form: August 10, 2015

published: October 14, 2015

Citation: Hudspeth J, El-Kareh R, Schiff G. Use of an Expedited Review Tool to Screen for Prior Diagnostic Error in Emergency Department Patients. Appl Clin Inform 2015; 6: 619-628

http://dx.doi.org/10.4338/ACl-2015-04-RA-0042 


\section{Background}

Missed or delayed diagnoses are an important area of medical error that result in significant morbidity and mortality $[1,2]$, and identifying and decreasing the rate of diagnostic error represents a major unmet quality improvement need. Claims for misdiagnosis are the leading cause of malpractice cases [3-7], but determining their true incidence remains difficult for multiple reasons. These include lack of agreement on definitions for diagnostic errors, or even whether these should be described more neutrally, as in "diagnostic discrepancies"; the subjective nature of determining whether a diagnosis could and should have been made earlier, with significant concerns regarding hindsight and outcome bias [8]; poor feedback systems, where diagnoses made in one encounter frequently are not connected to prior or future patient outcomes; the insensitivity of traditional approaches such as random case review, autopsy review, and malpractice claims in finding diagnostic error; and the typically time-intensive process of reviewing patient charts to identify diagnosis errors or delays [9]. This last barrier limits the extensive review of records in research, with one study of diagnostic errors reporting a mean review time of 1.4 hours per record [5]. An ED based study found that $86 \%$ of the diagnostic error uncovered were preventable, suggesting that errors of this nature could be reduced significantly, if not eliminated [10].

Recent studies harness the power of electronic health records (EHRs) by using "triggers" to filter data into subsets enriched with cases of diagnostic error, through selecting cases considered at higher risk for such errors [9-14]. By applying these screens to ambulatory EHRs they increased the prevalence of cases of error from $2.1 \%$ at baseline to $5.4 \%-20.9 \%$ in groups using screening triggers such as an unexpected return visit or hospitalization. In the Emergency Department (ED) context, return visits within 7 days had a rate of $5.7 \%$ adverse events, with $1.6 \%$ having adverse events from diagnostic errors [10], and about $20 \%$ of unscheduled returns had diagnostic errors [15].

One powerful and underutilized capability of electronic records is the capacity to rearrange information to cognitively support the process of assessing content in medical records. By optimizing display of longitudinal data, the EHR can be used facilitate comparison of "upstream" diagnostic assessments with "downstream" diagnostic outcomes [16, 17]. Motivated by findings from internal quality improvement programs, we designed an innovative rapid review tool combining our EHR and off the shelf software to expedite clinician review of medical records for diagnostic error, with the hypothesis that doing so would speed review. We combined this tool with a trigger to generate a retrospective case series of patients at potentially increased risk for diagnostic error -those with ED visits shortly after prior healthcare contacts- and then reviewed these cases for diagnostic errors, with secondary objectives of determining rates of error within this sample and exploring the utility of varying trigger criteria.

\section{Methods}

\subsection{Overview}

Using Partners Healthcare`s Longitudinal Medical Record (LMR), we identified all patients seen in our ED within a 31-day period spanning March 1, 2009 to March 31, 2009, and using electronic triggers identified the subset with any EHR note during the preceding 14 days. The EHR contained all outpatient and ED notes, all inpatient discharge summaries, and select inpatient notes. For the patient subset with any other EHR notes within the preceding 14 days of a sentinel ED visit, here defined as the ED visit within the specified period that triggered the case being pulled into our database, we imported all notes from 14 days before and 30 days afterwards into a Microsoft Access (Microsoft Corporation, Redmond, WA) database. The ED population was chosen due to our involvement in ongoing quality improvement efforts within that department, and due to a perception that diagnostic errors are an important problem in this clinical area [10, 15].

The database had a customized visual display to allow simultaneous comparison of the sentinel ED visit note to any other EHR notes within the specified range ( $>$ Figure 1 ). This tool was designed to enable the reviewing clinician to efficiently examine diagnostic assessments and outcomes from preceding visits, the sentinel ED visit, and any subsequent notes. The reviewer could thus evaluate 
the diagnostic accuracy of the earlier assessments relative to the ultimate diagnoses made during the sentinel ED visit and subsequent encounters.

A practice version of this Microsoft Access database with deidentified patient information is freely available on the journal website ( $\$$ Supplementary Material) or can be requested from the corresponding author.

The study ED is in an urban setting featuring multiple health systems, and is associated with a large academic medical center. Patient volume for the ED approaches 58000 visits per year.

\subsection{Review Process}

Each visit was rated on a drop-down menu with the probability of a missed or delayed diagnosis as "definite, probable, possible, unlikely, none," recognizing that missed or incorrect diagnoses are often difficult to categorize as absolutely present or absent. A designation of "incomplete" was used for records lacking sufficient information (e.g. patient elopement before clinician assessment, or a prior clinical visit that was an infusion or phone contact that did not involve direct contact with a clinician). Additional menus in the tool allowed specification of the type of missed or delayed diagnosis using a diagnosis error classification tool (DEER Taxonomy) [1] with a free text field for general comment on the cases; the classification tool was not used for this study.

All cases with the screen-positive database were reviewed by a general internist, who read the sentinel ED visit note as well as "upstream" and "downstream" visits' documents. We used a standard definition of diagnostic error (diagnosis "missed, wrong, or delayed, as detected by some subsequent definitive test or finding" [18] despite the physician having adequate information to make or suspect the ultimate diagnosis at the initial visit). As needed, the reviewer could access the full EHR for review for other data (labs, radiology) beyond that loaded into the rapid review tool. The initial reviewer put in a brief description of the case within the text field; they did not employ the DEER taxonomy portion of the tool for this study. A second general internist reviewed all of the "definite, probable, possible, and unlikely" cases, as well as a subset of "none" cases; disagreements were adjudicated by discussion to consensus. The number of cases requiring discussion or direct EHR review was not recorded. When a patient had multiple ED visits within the period, the reviewing physician decided if these related to a single diagnosis; patients could have multiple sentinel ED visits if each related to a separate diagnosis.

Time taken per session was recorded for the primary review, capturing the amount of time spent to review a series of cases rather than the individual cases themselves. Time spent by the second reviewer and discussion of disagreements in rating was not recorded. Prior to the review of the database a subset of 75 of these cases were chosen via random number generation and then reviewed by two general internists using the EHR alone to obtain the above information. The total time required to review this subset was recorded. These internists were not involved in the subsequent review of the total case series via the database tool.

\subsection{Statistical Analysis}

We calculated descriptive statistics including total ED visits, number of screen positive cases, the number of cases within different categories of likelihood, and number of incomplete cases. We performed a sensitivity analysis by varying the screening criterion of the number of days prior to the sentinel ED visits to search for preceding visits. For each value from 1 day to 14 days, we calculated the proportion of screen positive cases with the yield of identified diagnostic errors (positive predictive value) as well as the proportion of all identified diagnostic errors that were captured (sensitivity).

This study was approved by the Partners Healthcare IRB. 


\section{Results}

\subsection{Missed Diagnoses}

Of 5066 unique ED visits within the 31 day period studied, 1498 (30\%) had a potential prior ED, inpatient, or outpatient visit within the preceding 14 days. All of these screen positive records were reviewed; 77 cases (1.5\%) were excluded due to elopement of the patient during the sentinel ED visit or the actual absence of a clinical visit in the preceding 14 days.

Of the remaining 1421 cases, 9 were found to have "definite" missed or delayed diagnosis and 29 were judged "probable" diagnostic errors - we considered the combination of these categories to constitute the positive cases. One patient had two ED visits among those cases, both felt to be related to the same episode of care, giving a total of 37 individual cases (2.6\%) with "definite" or "probable" diagnostic errors.

An additional 42 cases (3\%) were "possible" cases of diagnostic error, with 58 cases (4.1\%) having some evidence of error but overall "unlikely" odds of being true diagnostic error; in the remaining cases $(1283 ; 90.3 \%)$ there was no evidence of a missed or delayed diagnosis. The range of missed or delayed diagnoses missed was broad ( $>$ Table 1), with 34 different diagnoses represented among the 37 positive cases, spanning relatively common pathologies to more unusual diseases. Pneumonia was the most frequent diagnosis, with three cases missed, constituting $8 \%$ of the missed diagnosis cases.

\subsection{Electronic Trigger Threshold}

Varying the threshold to trigger a review (i.e. the number of days prior to the sentinel ED visit the database searched for a preceding encounter) improved the percentage of cases positive for "definite" or "probable" errors at some cost to sensitivity ( $>$ Figure 2). For example, reducing the interval 14 days down to 4 days, the yield of positive cases improved from $2.7 \%$ to $3.8 \%$, but at a reduction in sensitivity to capturing only $71 \%$ of the positive cases.

\subsection{Review Time Required Using Tool}

Review of 1498 cases took a total of 2876 minutes, for a mean of 1.9 minutes per case; as we only timed the review of a group of cases at a time, rather than the time needed to review individual cases, we do not have information on the median or standard deviations. By comparison, review of a subset of the same cases using the EHR alone took 840 minutes for 75 charts (11.2 minutes/chart), representing an $83 \%$ decrease in time per case review.

The average number of notes in the database per reviewed case was 12.3 , with a median of 7 and maximum of 105 . Notes varied considerably in length, ranging from multi-page discharge summaries to single paragraph ED or phone notes.

\section{Discussion}

$30 \%$ of all patients seen in this urban emergency department had an EHR documented clinical visit during the preceding 14 days, and of these $2.7 \%$ had evidence of a definite or probable misdiagnosis, with another $7 \%$ having evidence of a possible misdiagnosis. As with prior studies $[11,14]$, the range of missed diagnoses was wide, containing both common and rare diagnoses. Unlike prior studies performed within a primary care setting the incidence of missed diagnoses within our targeted case series was relatively low, with only $2.7 \%$ of cases positive, as compared to an average population rate of $5.08 \%$ in a summative series combining several studies looking at outpatients [19]. Moreover, trials employing similar algorithms to enrich diagnostic errors found rates ranging 5.4-20.4\% [11, 12]. Potential contributors to this gap include differences in population between ED and primary care, with an ED based trial finding a diagnostic error rate of 1.6\% in ED revisits within 7 days [10], though many of our cases were first seen in a primary care clinic; differences between the healthcare systems of the different studies; and differences in criteria used to define a misdiagnosis as well as 
the degree of certainty required for giving that label. Towards this last point, if we expand our definition of a case to include "possible" cases as well as "definite and probable," our overall rate would become $5.6 \%$, which is more in keeping with the preceding literature. The lower percentage may also reflect the shorter time window (45 days) during which we examined the patient's care and course; if we reviewed more of the preceding and subsequent records, more diagnostic errors would likely emerge.

Combining electronic notes with a new dedicated review tool provided substantially more efficient chart review when screening for diagnostic errors. While the paper inpatient and ED notes were not reviewed, the full EHR records provided considerable information for adjudicating questions of missed diagnosis; as healthcare moves towards comprehensive EHR systems, this approach will capture an increasing amount of both the unstructured data as well as the clinical reasoning in clinician notes. Our tool provided a side by side view comparing the sentinel ED visit to other visits, thus streamlining the cognitive work of tracking patients' diagnostic assessments as they evolved over time. With one click subsequent "downstream" discharge summary assessments could be compared with "upstream" clinic or ED notes to track accuracy and outcomes. Our approach holds potential for efficient, large studies of diagnostic error that could assist both researchers studying the epidemiology of diagnostic error as well as institutions aiming to evaluate and reduce their rates of medical error. While we used an ED population for this study, the methods employed could readily be applied to other healthcare settings, though the speed of review will naturally correlate with the amount of records generated by each patient.

\subsection{Limitations}

There are several limitations to our study. We captured only visits recorded within this EHR system, thus overlooking prior or subsequent visits outside our network, in a city with multiple health systems; additional misdiagnosis cases were thus likely overlooked as outside records were not available, making our estimate of prevalence more conservative. Missed diagnoses with a longer delay between initial presentation and subsequent visits, including most indolent diseases, would not have been captured within our two-week period. Too, we were dependent on information and reasoning documented in the EHR, therefore falsely flagging instances where a clinician considered, but did not document, the ultimate diagnosis. Conversely, there may be missed diagnoses still missed on repeat presentations.

We also recognize that rating a missed or delayed diagnosis involves subjective judgment, and there are appreciable concerns that reviewers will consistently overestimate the degree to which a diagnosis was truly missed given the constraints of the initial interaction $[8,20]$. We attempted to control for this by using explicit criteria as well as having a second reviewer for all cases with any possibility of missed diagnoses, plus a random sample of the negative subset of cases. Prior reviews have found poor to fair inter-reviewer agreement [12], and thus this is likely a limitation for our findings as well, although our approach of a secondary review of all positive cases likely lowered the sensitivity of the review and the incidence in a conservative direction, particularly as not all negative cases were reviewed for false negatives. Review of cases by internists likely results in different determinations of diagnostic error than by emergency medicine physicians; as the majority of our positive cases were seen in outpatient clinics prior to their sentinel ED visit, rather than in the ED, we felt comfortable having internists review these cases.

The time spent per case depends heavily on the number of notes and the note length, and the notes from ED visits within our system are typically quite brief, so the speed with which review occurred in this study may not carry over settings with longer notes (e.g. inpatient settings). That said, we do feel that this approach would speed review of any record. We also acknowledge that the reviewers differed between the comparison case review (EHR only) and the review employing the database tool; we decided on this approach given that having the same reviewer for both case reviews could constitute a source of bias.

Finally, while our case set of patients with an ED visit shortly after preceding clinical interactions intuitively would be enriched for medical errors, we did not perform a control group review for the "trigger" aspect of our tool. It is possible that our selection process did not actually produce a subset 
enriched for missed or delayed diagnoses relative to the general population of patients visiting the ED. This would not impact the rapid review aspect of the study.

\section{Conclusions}

We leveraged the capabilities of an established EHR to build an innovative visualization and review tool to screen for cases of diagnostic errors in outpatient and ED visits. With the increasing use and size of EHRs, developing software that reconfigures data to allow for easier cognitive processing in review will be an important step in realizing the full potential of electronic records as instruments of quality improvement. This study adds to existing data on the approach of using triggers to identify high-risk patients (e.g. early return after preceding clinic or ED visit) to improve the yield of diagnostic errors, and our approach can be used with a range of criteria to target various types of diagnostic errors. Given that many of these errors are preventable, these data provide an opportunity for increased patient safety and quality improvement. Importantly, we demonstrate that the use of a simple software tool can significantly speed record review, providing a quantitative advance in efficiency that represents qualitative leap in the ability to screen for diagnostic errors.

\section{Clinical Relevance Statement}

Diagnostic errors are a large problem within healthcare that are difficult to accurately capture. This paper discusses using a software tool to expedite record review for diagnostic errors, potentially allowing future studies to review larger groups of patients for diagnostic errors through electronic health records.

\section{Conflict of Interest Statement}

The authors declare they have no conflicts of interests in this research.

\section{Human Subjects Protections}

The study was performed in compliance with the World Medical Association Declaration of Helsinki on Ethical Principles for Medical Research Involving Human Subjects, and was reviewed by the Partners Healthcare Institutional Review Board.

\section{Acknowledgements}

James Hudspeth received support from the Brigham and Women's Hospital Internal Medicine Residency Program; Robert El-Kareh and Gordon Schiff received support from CRICO/RMF. Robert El-Kareh was also supported by a National Library of Medicine informatics training grant (2 T15 LM007092).

\section{Contributorship Statement}

$\mathrm{JH}$ performed the data collection, drafted the paper, and revised the paper. He is the guarantor. RE-H designed the Access database tool, pulled the information from the Partners Healthcare EHR, analyzed the data, and revised the paper. GS reviewed collected data and revised the paper. GS and RE-H designed the study.

\section{Funding Statement}

This research received no specific grant from any funding agency in the public, commercial or notfor-profit sectors. 


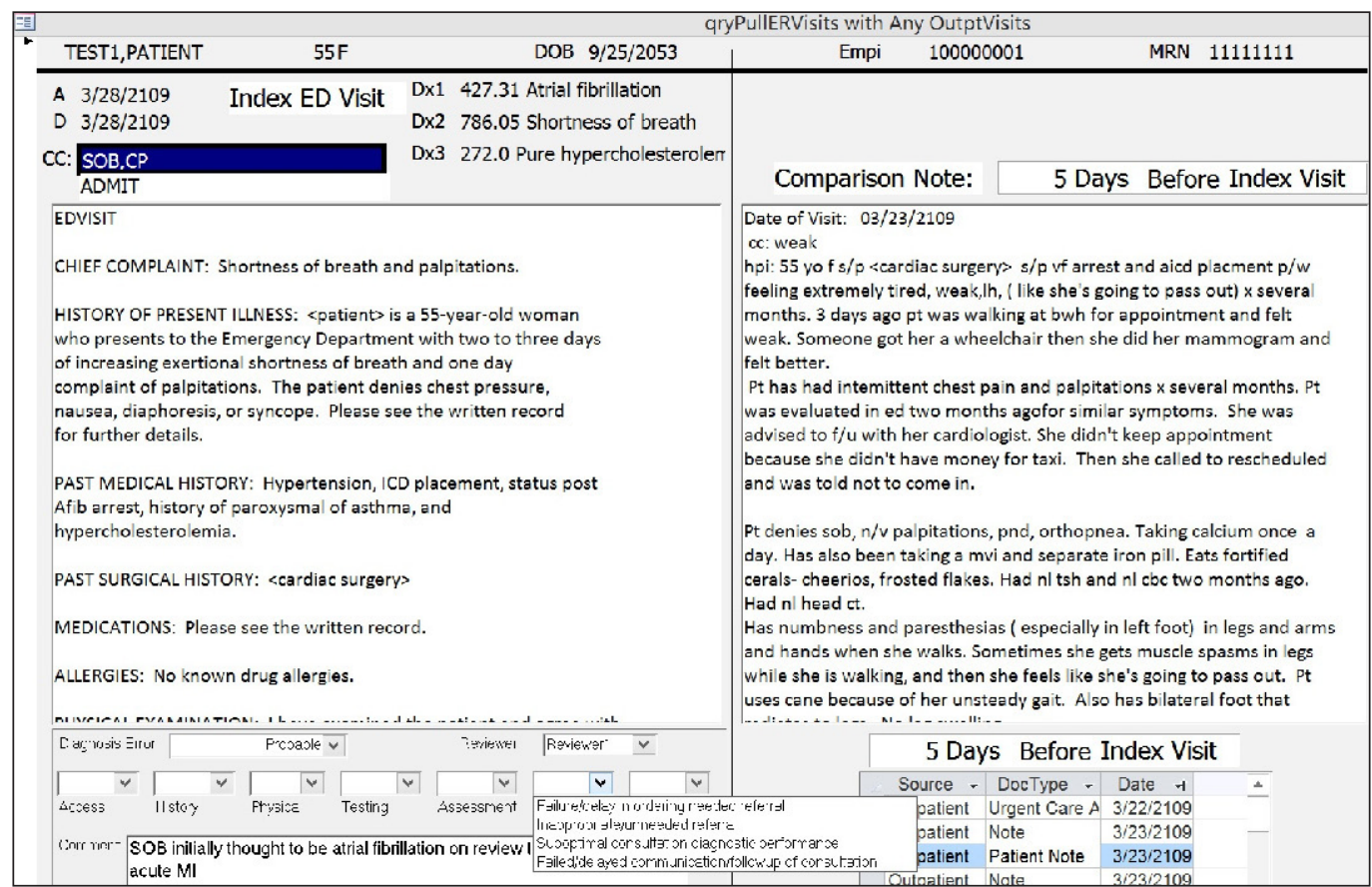

Fig. 1 Screenshot of the database visual display: this displays the index ED visit in the upper left next to a prior clinical document for review on the upper right; to the bottom right is a list of the available documents pulled into the database; and the bottom left holds an area for reviewer comments and ratings.

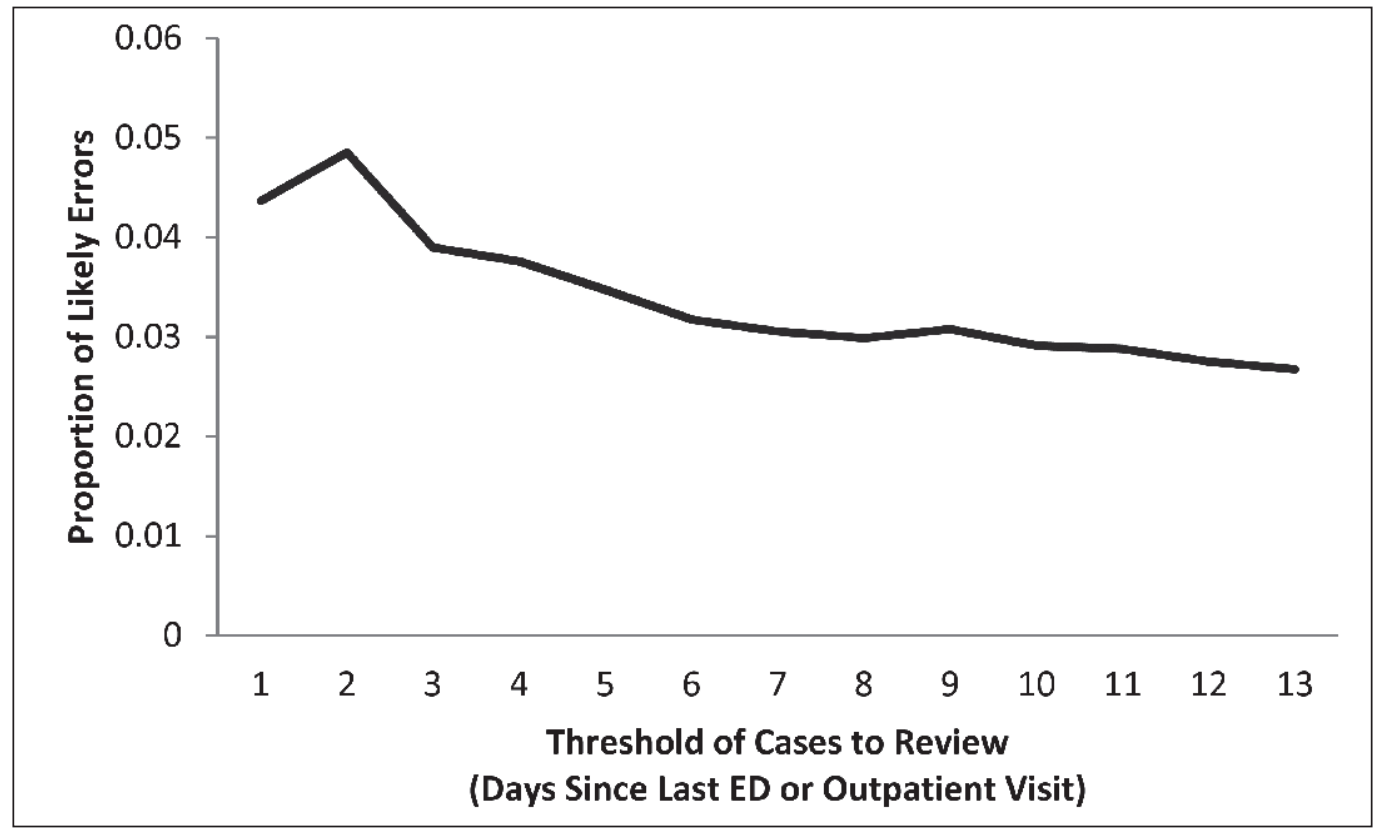

Fig. 2 Impact of varying the threshold for triggering review: this shows the change in the proportion of reviewed cases positive for diagnostic error when varying the threshold of days before the index ED visit that triggered review. 
Table 1 List of the missed diagnoses, grouped by category and including the initial diagnosis.

\begin{tabular}{|c|c|c|c|c|}
\hline Age & Preceding Contact & Initial Diagnosis & Final Diagnosis & Time to ED Visit \\
\hline 57 & Inpatient & post-operative pain & $\begin{array}{l}\text { post-operative epidural } \\
\text { abscess }\end{array}$ & 7 days \\
\hline 51 & Orthopedics & Seroma & surgical site infection & 1 day \\
\hline 58 & Primary Care & low back pain & pyelonephritis & 1 day \\
\hline 45 & ED & gum infection & dental abscess & 1 day \\
\hline 19 & Rheumatology & neck strain & $\begin{array}{l}\text { retrophyayrngeal } \\
\text { abscess }\end{array}$ & 1 day \\
\hline 45 & Gynecology & drug related diarrhea & C. difficile colitis & 2 days \\
\hline 32 & Primary Care & Fistula & perirectal abscess & 2 days \\
\hline 63 & Pulmonary & ILD flare & pneumonia & 4 days \\
\hline 40 & Urgent Care & $\begin{array}{l}\text { upper respiratory } \\
\text { infection }\end{array}$ & pneumonia & 2 days \\
\hline 65 & Oncology & $\begin{array}{l}\text { upper respiratory } \\
\text { infection }\end{array}$ & pneumonia & 4 days \\
\hline 78 & Oncology & $\begin{array}{l}\text { community acquired } \\
\text { pneumonia }\end{array}$ & $\begin{array}{l}\text { healthcare associated } \\
\text { pneumonia }\end{array}$ & 1 day \\
\hline 80 & Vascular Medicine & no clear diagnosis made & pleural effusion & 4 days \\
\hline 59 & Oncology & Anemia & pleural effusion & 6 days \\
\hline 68 & Rheumatology & dermatomyositis flare & congestive heart failure & 4 days \\
\hline 55 & Primary Care & $\begin{array}{l}\text { electrolyte abnormality } \\
\text { vs neuropathy }\end{array}$ & atrial fibrillation & 5 days \\
\hline 32 & Oncology & pneumonia & cardiomyopathy & 8 days \\
\hline 45 & ED & mild bleeding & severe uterine bleeding & 7 days \\
\hline 40 & Gynecology & endometriosis & $\begin{array}{l}\text { retained products of } \\
\text { conception }\end{array}$ & 2 days \\
\hline 63 & Urgent Care & $\begin{array}{l}\text { abdominal pain/nausea, } \\
\text { unclear source }\end{array}$ & kidney infarction & 2 days \\
\hline 65 & Oncology & post-operative swelling & deep vein thrombosis & 5 days \\
\hline 90 & Primary Care & urinary tract infection & lung cancer & 16 days \\
\hline 49 & ED & $\begin{array}{l}\text { hypertension related } \\
\text { headache* }\end{array}$ & Call-Fleming syndrome & 5 days \\
\hline 19 & ED & neck strain & migraine & 4 days \\
\hline 28 & Primary Care & pyelonpehritis & sciatica & 2 days \\
\hline 72 & Oncology & $\begin{array}{l}\text { urinary frequency from } \\
\text { glucosuria }\end{array}$ & $\begin{array}{l}\text { severe spinal stenosis } \\
\text { with cystitis }\end{array}$ & 2 days \\
\hline 54 & ED & status migrainous & temporal lobe seizures & 1 day \\
\hline 66 & Primary Care & cervical/lumbar strain & myositis & 6 days \\
\hline 26 & Rheumatology & social stress & lupus flare & 1 days \\
\hline 88 & Primary Care & Cystitis & hyponatremia & 4 days \\
\hline 77 & ED & Bursitis & gout flare & 3 days \\
\hline 32 & ED & Trauma & foreign body in ear lobe & 1 day \\
\hline 56 & Urgent Care & $\begin{array}{l}\text { increased intracranial } \\
\text { pressure }\end{array}$ & corneal irritation & 1 day \\
\hline
\end{tabular}


Table 1 Continued

\begin{tabular}{|l|l|l|l|l|}
\hline Age & Preceding Contact & Initial Diagnosis & Final Diagnosis & Time to ED Visit \\
\hline 19 & ED & $\begin{array}{l}\text { upper respiratory } \\
\text { infection }\end{array}$ & allergic reaction & 1 day \\
\hline 56 & ED & venous insufficiency & ankle fracture & 1 day \\
\hline 51 & Oncology & abdominal gas & ascites & 3 days \\
\hline 19 & Urgent Care & Bursitis & Baker's cyst & 9 days \\
\hline 28 & ED & pneumonia & $\begin{array}{l}\text { post operative biliary } \\
\text { leak }\end{array}$ & 2 days \\
\hline
\end{tabular}

* this patient was seen twice within the database, and both cases were judged due to the same ultimate diagnosis 


\section{References}

1. Schiff GD, Kim S, Abrams R, Cosby K, Lambert B, Elstein AS, Hasler S, Krosnjar N, Odwazny R, Wisniewski MF, McNutt RA. Diagnosing Diagnosis Errors: Lessons from a Multi-institutional Collaborative Project. In: Henriksen K, Battles JB, Marks ES, Lewin DI, eds. Advances in Patient Safety: From Research to Implementation (Volume 2: Concepts and Methodology). Rockville, MD: Agency for Healthcare Research and Quality (US) 2005; 255-278.

2. Newman-Toker DE, Pronovost PJ. Diagnostic errors: the next frontier for patient safety. JAMA 2009; 301: 1060-1062.

3. Gandhi TK, Kachalia A, Thomas EJ, Puopolo AL, Yoon C, Brennan TA, Studdert DM. Missed and delayed diagnoses in the ambulatory setting: a study of closed malpractice claims. Ann Intern Med 2006; 145: 488-496.

4. Studdert DM, Mello MM, Gawande AA, Gandhi TK, Kachalia A, Yoon C, Puopolo AL, Brennan TA. Claims, errors, and compensation payments in medical malpractice litigation. N Engl J Med 2006; 354: 2024- 2033.

5. Kachalia A, Gandhi TK, Puopolo AL, Yoon C, Thomas EK, Griffey R, Brennan TA, Studdert DM. Missed and delayed diagnoses in the emergency department: a study of closed malpractice claims from 4 liability insurers. Ann Emerg Med 2007; 49: 196-205.

6. Saber Tehrani AS, Lee H, Mathews SC, Shore A, Makary MA, Pronovost PJ, Newman-Toker DE. 25-Year summary of US malpractice claims for diagnostic errors 1986-2010: an analysis from the National Practitioner Data Bank. BMJ Qual Saf 2013; 22: 672-680.

7. Schiff GD, Puopolo AL, Huben-Kearney A, Yu W, Keorhan C, McDonough P, Ellis BR, Bates DW, Biondolillo M. Primary care closed claims experience of Massachusetts malpractice insurers. JAMA Intern Med 2013; 173: 2063-2068.

8. Wears RL, Nemeth CP. Replacing hindsight with insight: towards a better understanding of diagnostic failures. Ann Emerg Med 2007; 49(2): 206-209.

9. Graber ML. The incidence of diagnostic error in medicine. BMJ Qual Saf 2013; 22: ii21-ii27.

10. Calder L, Pozgay A, Riff S, Rothwell D, Youngson E, Mojaverian N, Cwinn A, Forster A. Adverse events in patients with return emergency department visits. BMJ Qual Saf 2015; 24(2): 142-148.

11. Singh H, Thomas EJ, Khan MM, Petersen LA. Identifying diagnostic errors in primary care using an electronic screening algorithm. Arch Intern Med 2007; 167: 302-308.

12. Singh H, Giardina TD, Forjuoh SN, Reis MD, Kosmach S, Khan MM, Thomas EJ. Electronic health record-based surveillance of diagnostic errors in primary care. BMJ Qual Saf 2012; 21: 93-100.

13. Schiff G. Finding and fixing diagnosis errors: can triggers help? BMJ Qual Saf 2012; 21: 89-92.

14. Singh H, Giardina TD, Meyer AN, Forjuoh SN, Reis MD, Thomas EJ. Types and origins of diagnostic errors in primary care settings. JAMA Intern Med 2013; 173: 418-425.

15. Nuñez S, Hexdall A, Aguirre-Jaime A. Unscheduled returns to the emergency department: an outcome of medical errors? Qual Saf Health Care 2006; 15: 102-108.

16. Plaisant C, Mushlin R, Snyder A, Li J, Heller D, Shneiderman B. LifeLines: using visualization to enhance navigation and analysis of patient records. Proc AMIA Symp 1998: 76-80.

17. Bui AA, Aberle DR, Kangarloo H. TimeLine: visualizing integrated patient records. IEEE Trans Inf Technol Biomed 2007; 11: 462-473.

18. Graber M. Diagnostic errors in medicine: a case of neglect. Jt Comm J Qual Patient Saf 2005; 31: 106-113.

19. Singh H, Meyer AN, Thomas EJ. The frequency of diagnostic errors in outpatient care: estimations from three large observational studies involving US adult populations. BMJ Qual Saf 2014; 23: 727-731.

20.Henriksen K. Improving diagnostic performance: some unrecognized obstacles. Diagnosis 2014; 1(1): 35-48. 